\title{
THERMOVISION IN THE VALIDATION PROCESS OF NUMERICAL SIMULATION OF BRAKING
}

\author{
Pawel Baranowski ${ }^{1)}$, Krzysztof Damaziak ${ }^{1)}$, Jerzy Malachowski ${ }^{1}$, Lukasz \\ Mazurkiewicz $^{1)}$, Henryk Polakowski²), Tadeusz Piatkowski ${ }^{2)}$, Mariusz Kastek ${ }^{2)}$ \\ 1) Military University of Technology, Faculty of Mechanical Engineering, Department of Mechanics and Applied Computer Science, Gen. \\ S. Kaliskiego 2, 00-998 Warsaw, Poland ( $₫$ pbaranowski@wat.edu.pl, +48 $22 \quad 683 \quad 9683, \quad$ kdamaziak@wat.edu.pl, \\ jerzy.malachowski@wat.edu.pl,lmazurkiewicz@wat.edu.pl). \\ 2) Military University of Technology, Institute of Optoelectronics, Gen. S. Kaliskiego 2, 00-998 Warsaw, Poland \\ (凹 hpolakowski@wat.edu.pl, +48 22683 9383,tpiatkowski@wat.edu.pl, mkastek@wat.edu.pl)
}

\begin{abstract}
This article presents the validation process of a brake FE model by means of temperature measured on a special stand using infrared technology. Unlike many other publications, the authors try to show the interaction between measurement technology and numerical modeling rather than only nice, perfectly correlated graphs. Some difficulties in choosing and using validation parameters are also pointed out and discussed. Finally, results of FE analyses are compared with measured data, followed by explanation of applied numerical technology and estimation of validation process effectiveness.
\end{abstract}

Keywords: thermovision, numerical models validation, temperature measurement.

\section{Genesis of the problem}

The investigations presented in the paper were part of a larger project whose goal was to design a new brake for a terrain vehicle. They covered the measurement of the brake lining wear process conducted on a special stand and trial called IL-68 (Fig. 1) and reproduction of these tests using numerical simulations [1].

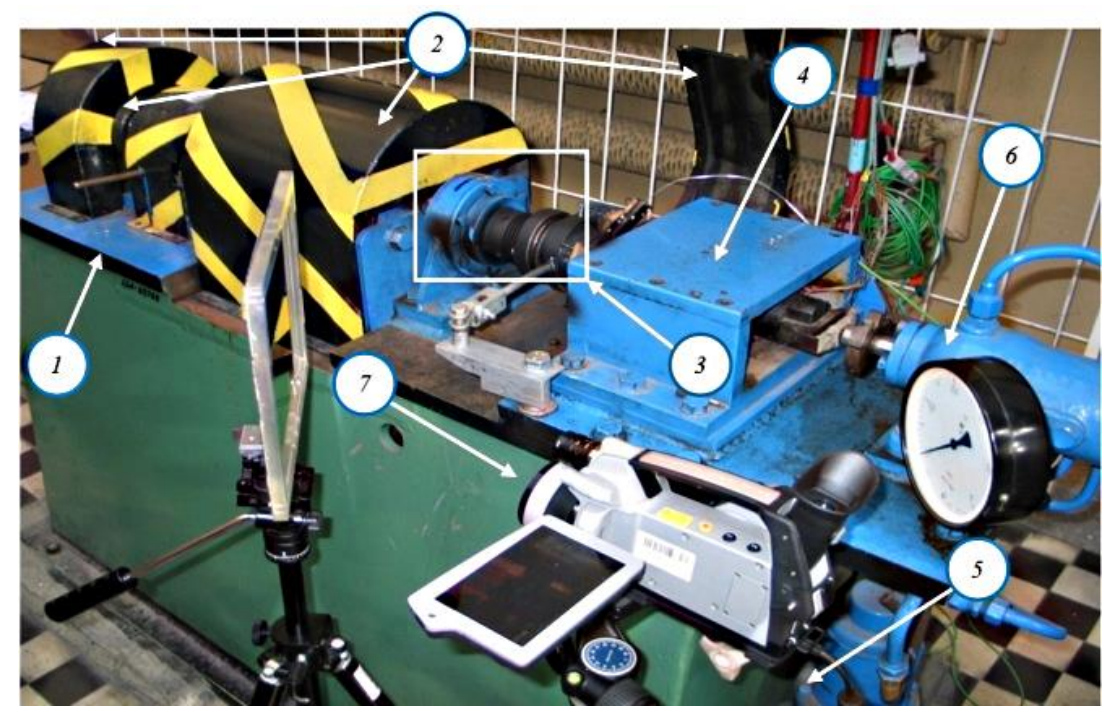

Fig. 1. Special stand IL-68: 1) Base 2) Rotating parts hoods, 3) Friction pair, 4) Press,

5) Water pump supplying the cooling subsystem, 6) Manometer, 7) Infrared camera. 
The latter was driven by the simple fact that numerical analysis gives far more information about the investigated process than measurements taken during tests. The assumption behind the above statement is that an analysis actually describes the phenomenon under investigation and enables its proper testing. Such correlation should be established by proper validation of the numerical model.

Basically, IL68 consists of a rotating part with so-called counter-sample mounted in it, which is acting as brake disk and a head pushing against the counter-sample, where lining samples are mounted (Fig. 2). Originally the special stand enabled the measurement of such quantities as angular velocity of the rotating part, the moment generated by friction forces, braking time and wear of lining defined as its thickness reduction. All these data were useful enough to empirically select lining best suited for a certain vehicle, but their use for validating numerical models was associated with at least two drawbacks, which will be explained in the section describing the choice of validation parameters.

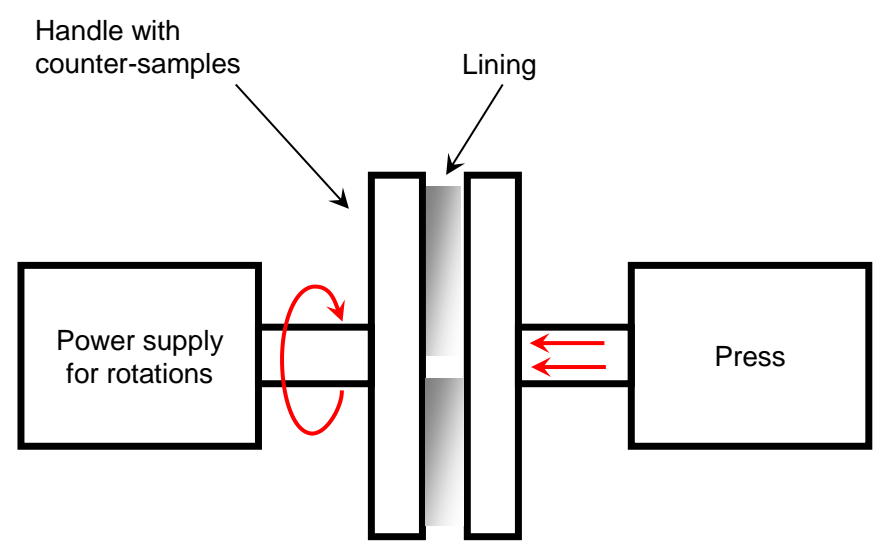

Fig. 2. Schematic describing the special stand work principle.

\section{Numerical model - assumptions and brief description}

Hands-on experience shows that the braking process generates heat. In reference [2] the author has stated that $95 \%$ of work done by friction forces is converted to heat. Additionally, it can be observed that a heat exchange process, as well as change in kinetic energy is nonstationary and rather short in time. The above observations lead to the conclusion that in numerical analysis thermo-mechanical coupling and heat generation by friction force cannot be neglected and the process should be analyzed as a dynamic, short-duration event. The authors decided that a suitable numerical tool of choice will be LS-Dyna, which is based on the Finite Element Method (FEM) and is dedicated to simulate short time-events and allows analyzing a so-called weak thermo-mechanical coupling. Such type of coupling means that the mechanical part described by equation (1) and thermal part of the problem (see equation 2) are solved independently. Coupling is achieved through the exchange of data between mechanical and thermal time steps where both solvers achieve a solution. The mechanical solver takes information about temperature distribution and based on it evaluates material data and updates the displacement field, while the thermal solver takes data about the displacement field:

$$
\int_{t_{p}}^{t_{k}}\left[\mathbf{U}^{T} \mathbf{M} \mathbf{U}+\mathbf{U}^{T} \mathbf{K} \mathbf{U}-\mathbf{F}^{T} \mathbf{U}\right] d t=0,
$$

where: $\mathbf{M}$ - mass matrix, $\mathbf{K}-$ stiffness matrix, $\mathbf{U}-$ displacement vector, $\mathbf{F}-$ external load vector, $t$ - time. 
The thermal part of the problem is solved based on the following equation:

$$
\mathbf{C} \frac{d \mathbf{T}}{d t}+\mathbf{K}_{p} \mathbf{T}=\mathbf{R}
$$

where: $\mathbf{C}$ - capacitance matrix, $\mathbf{K}_{p}$ - conductivity matrix, $\mathbf{R}$ - heat sources vector, $t$ - time.

In the chosen numerical tool, equation (1) is solved using central difference explicit algorithm, while equation (2) is solved using implicit method.

A few test models were developed in order to verify and properly choose such important aspects of numerical modeling as contact algorithm, thermal-mechanical coupling or element formulations. A detailed description of one of such tests can be found in [3]. The resultant FE model of a special stand is presented in Fig. 3.

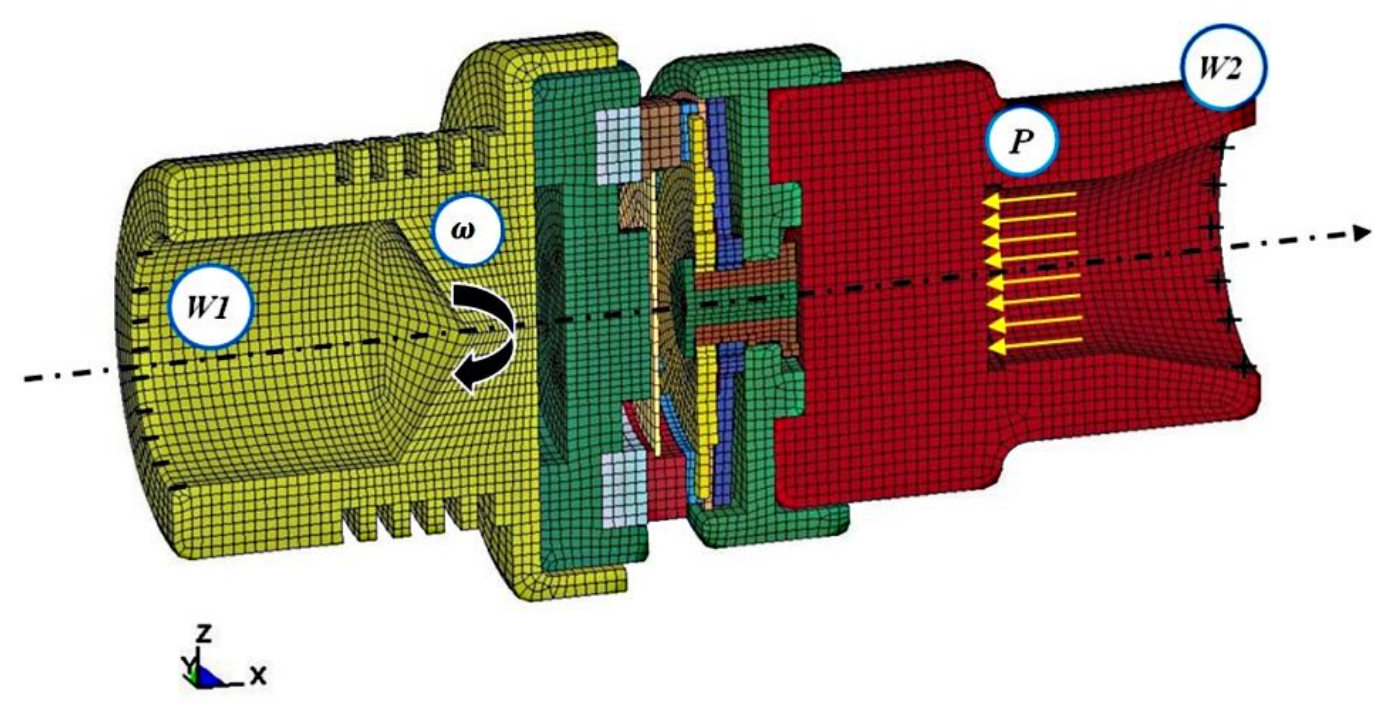

Fig. 3. Half of the FE model of the special stand: rotating (W1) and pushing (W2) parts are marked [1].

The FE model consisted of 88519 elements and 109247 nodes. The initial integration time step of the mechanical part of the problem has stabilized on the value of $\Delta t=5.65 \cdot 10^{-8} \mathrm{~s}$. With such a short time step it was virtually impossible to cover the typical braking time observed during tests, which was about $10 \mathrm{~s}$, since numerical analysis of the first second took a few weeks running on $8 \mathrm{CPU}$ units.

It should be noted that the nature of the analyzed phenomenon does not allow the use of axial symmetry. Moreover, the fact that large rotation is involved in the process, the application of different integration algorithms (e.g. implicit ones) would not shorten run times significantly. The only simplification present in the model was the assumption that there is no heat convection between the friction pair (counter-samples and lining) and the rest of the stand.

The other way to make the model smaller would be to remove some parts of the stand. Unfortunately, this approach would not be effective in case of IL-68. Explicit algorithms are not as sensitive to model size as implicit ones, showing only linear dependence on this parameter. This would mean that reducing the number of equations by half would give a model which would still require days and not hours to analyze. 


\section{Choice of validation parameters}

Philosophers claim that the term "validation" is used by scientists in very misleading way. They propose term "confirmation" instead. Nevertheless, the term "validated" used as a statement of the fact that mathematical (or numerical) model prediction consisted with observational data is so widely used that authors stick to this nomenclature [4]. Whatever the name of the process should be, the goal of it is still the same and it should start with choice of parameters allowing to prove the mentioned consistency.

Properly chosen validation parameters should have the following characteristics:

- should allow direct comparison of results taken from experiment against computed ones,

- the acquisition process of experimental data should give clear and straightforward output so that the data will require simple post-processing and leave no room for interpretations,

- compared parameters should allow the verification of most important (for given case) numerical algorithms and not only trivial, most basic ones.

All this leads to the conclusion that the original IL-68 stand did not provide data good enough for a proper validation process. As mentioned before, the stand allowed the measurement of a few parameters describing braking properties of the lining material. Nevertheless, besides wear, all measured parameters were - in fact - a simple function of friction force. This means that measured quantities could be used to validate the most basic parameters of the numerical model only, including proper mass and dimensions (inertia) definition, together with proper load transition, which turns into validation of initial and boundary conditions of the mechanical part of simulation. In particular, these parameters did not allow a validation of the thermo-mechanical coupling.

The other measured parameter (wear), is rather difficult to model numerically. This comes from the fact that wear takes place in micro scale [5,6], implying that detailed numerical simulation of the process should also be performed at the same level of modeling [7]. This implication comes from the fact that currently available numerical techniques are not ready for multiscale analyses. Therefore a numerical model of the whole stand can only use a parametric description of wear [8,9]. Unfortunately, despite the fact that there are plenty of such relations (the authors of review [10] found more than 300 of them), none of them is widely accepted and each of them requires additional, tribological tests due to the semiempirical nature of such formulas. Another problem with wear as validation parameter was related to the analysis run times. In order to cover total braking time using the FE model described above it required months of CPU time, while validation could only be done at the end of the analysis. This was simply not feasible.

Taking into account all above, the authors decided that the best quantity for comparison of numerical model against stand tests would be temperature. It would allow checking more advanced and crucial aspects of the FE model, like thermo-mechanical coupling and conversion of mechanical work into heat. It would also show the accuracy of contact modeling (friction force value) since in the FE model the amount of heat is linearly dependent on this force, according to the following formula:

$$
F_{t} v_{0}=c_{p} m \frac{\Delta T}{\Delta t}
$$

where: $F_{t}$ - friction force, $v_{0}-$ velocity, $c_{p}$ - heat capacity, $m$ - mass, $T$ - temperature, $t$ - time. 
It would be also possible to compare numerical and experimental data from the very beginning of the process and, last but not least, it was fairly easy to adapt the original IL-68 stand for such measurements. The only drawback of the chosen solution was the fact that temperature measurements on the modified stand were not straightforward, what will be shown in the next section.

It should be noticed once again, that in equation (2) radiation and convection are skipped in order to speed up the integration process. In so far as omission of radiation is not a big derogation from the actual conditions, the lack of convection is a rather big simplification $[11,12,13]$. It followed the assumption that at the very first moment of braking, convection can also be neglected due to the thermal inertia of the system.

\section{Temperature measurements}

It was decided that temperature will be measured using two different techniques: with thermocouples and by a thermovision camera. The decision was driven by the fact that the most critical area, where heat generation takes place, is on the lining surface. It was impossible to place thermocouples on this surface, but it was possible to make a hole in the lining housing so that the camera could "see" the surface of the counter-sample. The schematic of the set-up is presented in Fig. 4.

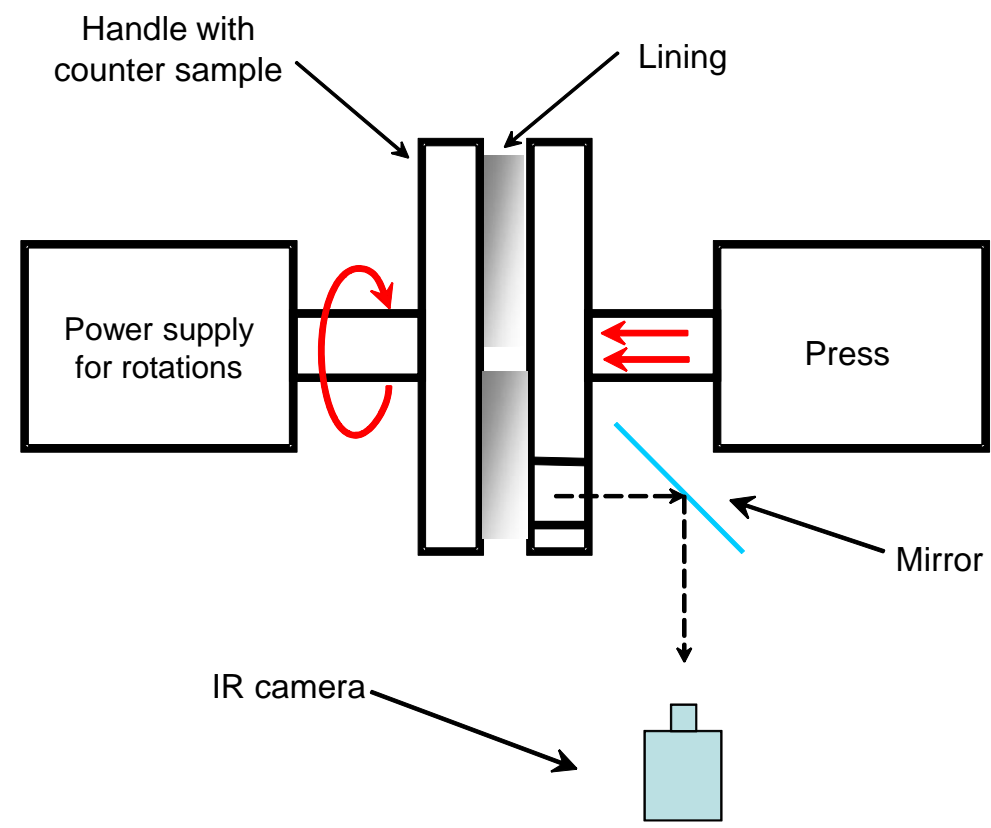

Fig. 4. Schematic showing the IR camera setup principle.

Due to lack of space for the IR camera in the axis of the measurement window and to protect the lens against wear products a secondary mirror was used in the stand. This was an optical-type mirror commonly used in infrared measurements, with the outer layer made of gold, having a reflection coefficient greater than $0.99 \mathrm{~m}$ in the spectral range between 3 and $5 \mu \mathrm{m}$. The mirror reversed the image, but it was irrelevant for the measurement. Taking into account other sources of errors, the mirror influence on the registration results can be neglected.

IR measurements were conducted in two stages. First the physical properties of brake pad linings (essential for further temperature measurements) were tested under laboratory conditions and then the whole brake was tested on the IL-68 test stand. 


\subsection{Thermal measurements of brake pad lining}

Brake pad linings are usually made of composite materials. During the tests the lining made from 500/EBC material type were used. The pads were first ground-in to obtain a constant braking force. The process of grinding-in the interacting surfaces consisted of several braking cycles in order to obtain an even, uniform surface of all tested pads. Subsequently, thermal uniformity of particular pads was verified. The whole pad module was evenly heated up and the temperature distribution in the stationary state across the pad surface was registered by a thermal camera. A sample thermal image of a brake lining surface is shown in Fig. 5a.

a)

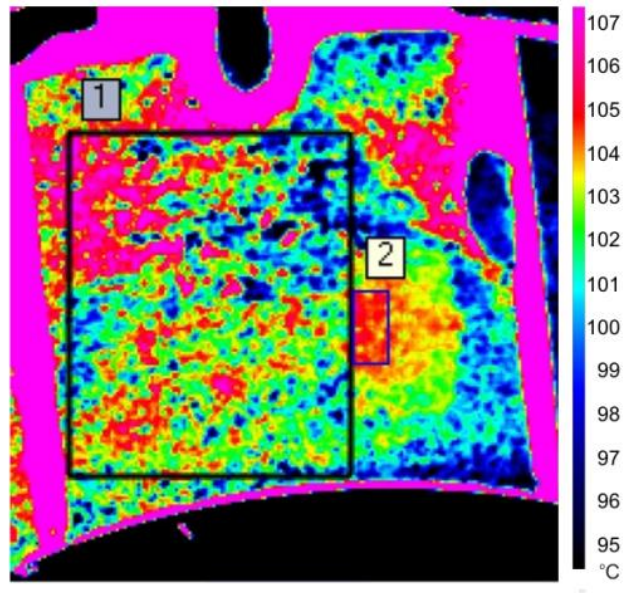

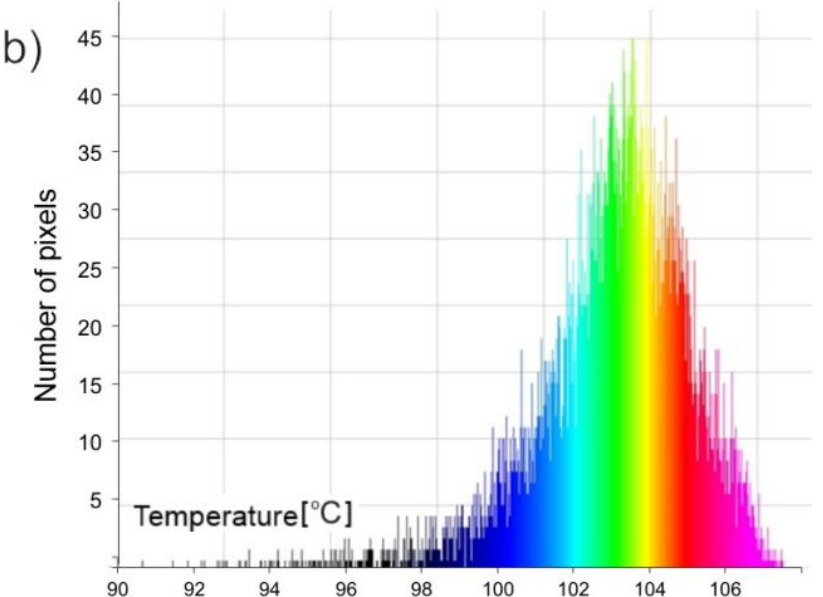

Fig. 5. Lining section, a) Temperature distribution on the surface, b) Temperature distribution on area 1.

Two areas were selected for temperature analysis. Area no. 2 is the reference one, covered with high-temperature paint of known emissivity. Lining-specific data were analyzed in area no. 1. Considerable temperature differences can be observed on the surface of a braking pad. The histogram of temperature data is depicted in Fig. 5b, which shows the number of points recorded at the given temperature. Observed temperature differences originate both from nonuniformity of the lining material and its surface structure. Composite materials are not uniform and they exhibit low thermal conductivity compared to a metal brake disk. As a result the temperature equalization does not occur in both the volume and on the surface of the composite material. The grinding-in process resulted in a mostly smooth braking surface. On such smooth fragments the recorded temperature is $T_{o_{-} a v}=103.8^{\circ} \mathrm{C}$ (corrected real value $T_{a v}=104.6^{\circ} \mathrm{C}$ ). However in the top left corner of the area 1 the recorded temperature value $T_{o} \geq 106^{\circ} \mathrm{C}$ (after correction $T_{o} \geq 105.3^{\circ} \mathrm{C}$ ) was observed. It is not the real temperature value, but is recorded as such due to higher effective emissivity of micro-cavities and cumulated wear remains. The effective emissivity of the surface is increased in this region up to $\varepsilon_{\text {effect }} \approx 1.0$. Direct measurements of the temperature of brake pad lining could then lead to significant measurement errors of $\delta T \approx \pm 10^{\circ} \mathrm{C}$ [14], due to the aforementioned in-depth and surface non-uniformity of the material structure.

The temperature of brake pad lining cannot be measured on the test stand during the braking cycle. It is only possible after the brake is halted and the two elements of the measurement head are separated. Then the brake pad lining can be introduced into the field of view of the thermal camera. As a result only relative temperature distributions can be recorded, because during the whole process the sample is cooled down before it can be 
visualized by a thermal camera. A sample temperature distribution recorded for a single braking pad element is shown in Fig. 6.

Below the temperature plot the thermal image is presented, showing the line along which the temperature profile was determined. $\mathrm{X}$-axis values are relative distances measured along this line from left to right. Considerable differences in temperature distributions can be observed between different brake pads as well as within a single pad area. Such behavior within a single pad could be expected on the basis of preliminary measurements, even in the quasi-stationary state after the full stop and separation of brake pads and the disk. However the differences between pads result from different braking forces applied to them and they should not be as evident as they are because each pad was adjusted and a running-in procedure was performed prior to measurements.

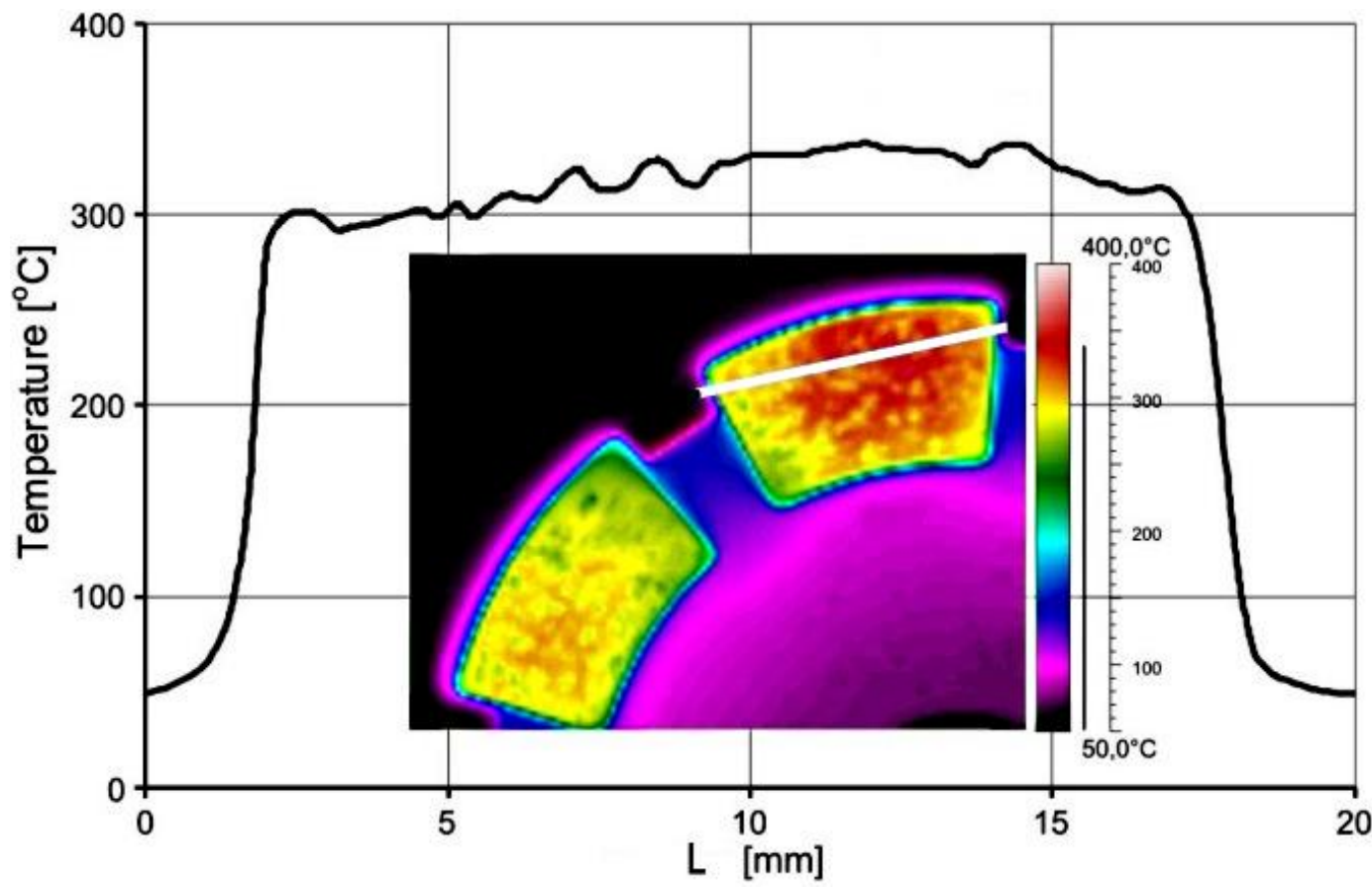

Fig. 6. Temperature distribution across a braking pad (white line) after a braking cycle.

\subsection{Comparative temperature measurements}

Usually thermocouples were applied to measure the temperature of brake elements. Those sensors were placed just under the surface of a braking pad. During the presented measurement sessions this classic approach was also used alongside non-contact temperature measurements by a thermal camera. A comparison of measurement results is graphically presented in Fig. 7 [15]. 


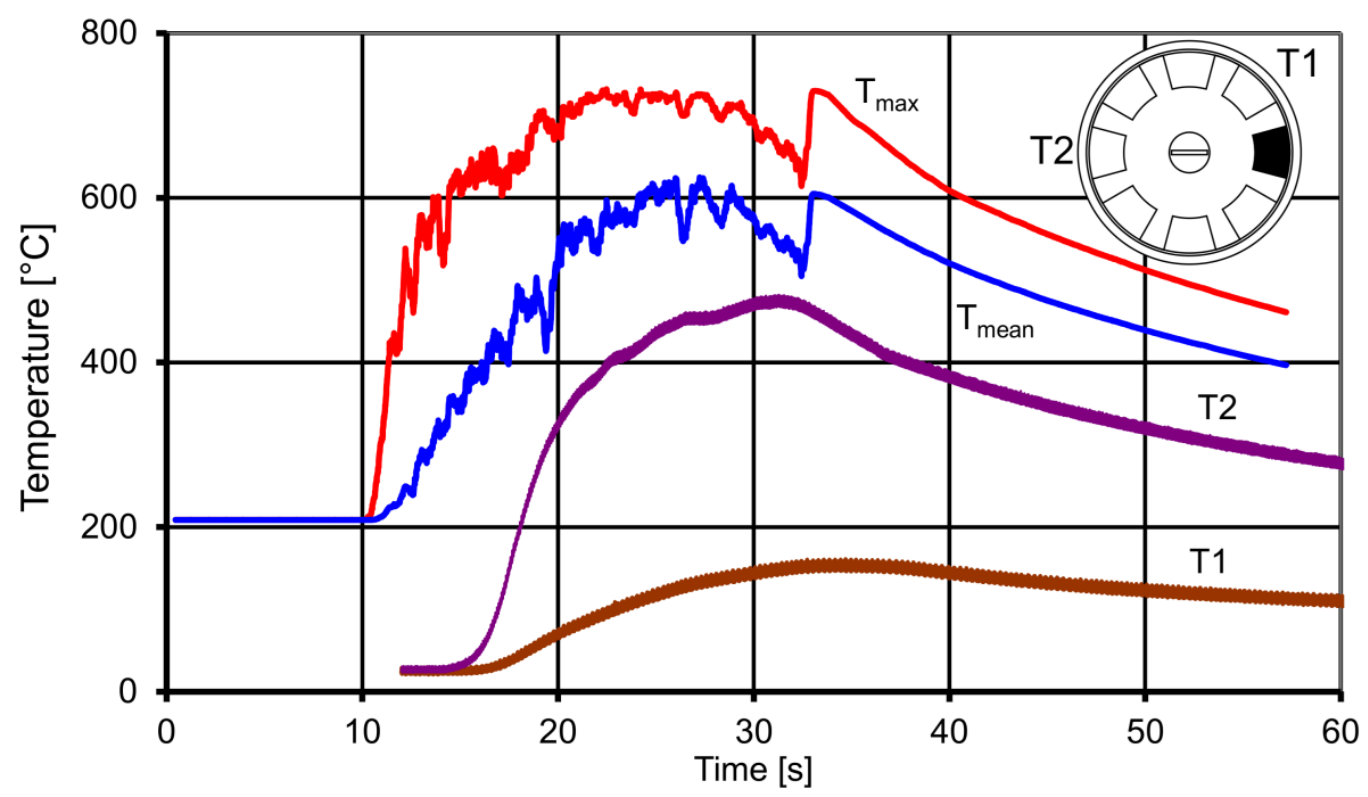

Fig. 7. Temperature changes inside a brake with time.

The positions of two thermocouples used to measure the temperatures of two brake pad segments $T_{1}$ and $T_{2}$ are schematically marked in the picture in the top-right corner. The black spot indicates the removed braking pad, which created the opening for the measurement of brake disc temperature by a thermal camera ( $T_{\max }$ and $\left.T_{\text {Mean }}\right)$. Due to high initial rotational speed $(6000 \mathrm{rpm})$ and consequently high linear velocity values, the data acquisition rate of the thermal camera was set at $200 \mathrm{fps}$.

It was assumed that the temperature of the contact area between the brace disk and pad is identical on both these interacting elements, thus by measuring the disk also the temperature of the pad can be assessed. Braking was started 8 seconds after the initialization of acquisition of temperature data. Due to limited dynamic range of the thermal detector it could only record temperature values in the $200^{\circ} \mathrm{C} \leq T \leq 710^{\circ} \mathrm{C}$ range. Previous measurements indicated that the expected temperature will not exceed $T_{\mathrm{b}} \leq 500^{\circ} \mathrm{C}$. And indeed, thermocouple measurement gave results below that threshold. However the thermal camera recorded considerably higher values, and after the 12-th second of the experiment the camera measurement range for preset exposure parameters was exceeded. Observed substantial differences between temperature readings from thermocouples $T_{1}, T_{2}$ and the thermal camera may result from uneven contact between particular brake pads and the brake disc. This occurred in spite of adjustments and running-in of the whole brake unit prior to the experiment.

\subsection{Thermal camera measurements of dynamic temperature changes}

Braking is a complex mechanical and thermodynamic process. Contact between the friction pair (pad and disc) is initially achieved locally and full contact of interacting surfaces takes place only in the last phase. This can be seen in the temperature distributions recorded during braking. Additionally as a result of using a slotted brake disc the camera records the thermal images of areas with very different temperatures. The sequence of thermal images of a disc brake recorded during a braking cycle is shown in Fig. 8. The initial rotational velocity was $6000 \mathrm{rpm}$ with the moment of inertia of the rotating masses set to $0.412 \mathrm{~kg} \cdot \mathrm{m}^{2}$, simulating the energy dissipation similar to the energy dissipated in the real brake, and the force acting on the friction pair was set to $5.00 \mathrm{kN}$. 

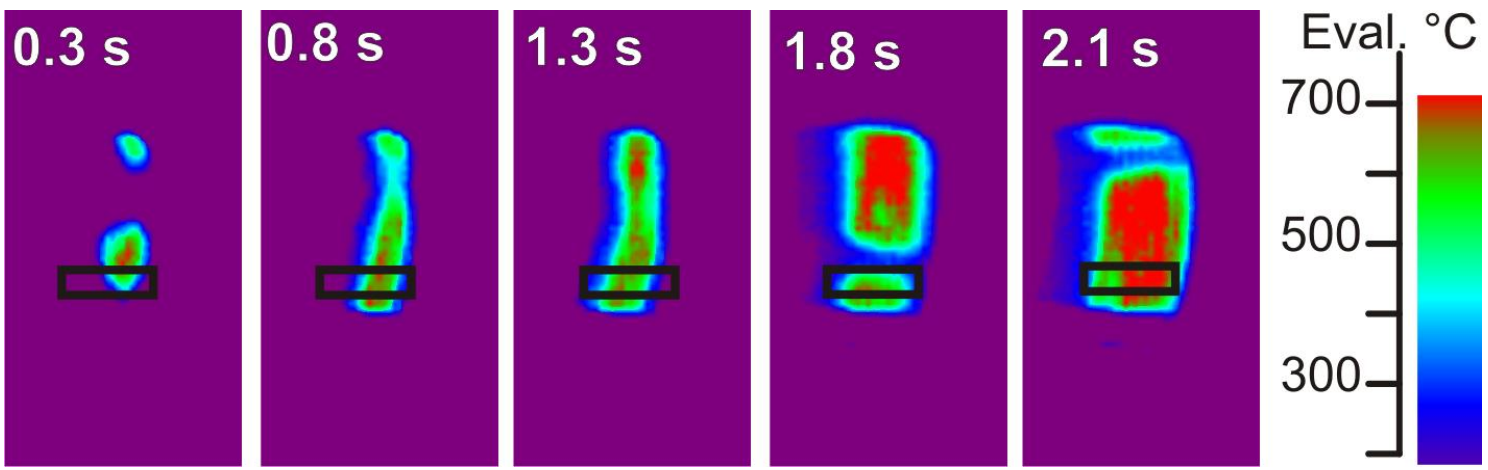

Fig. 8. Thermograms of counter-sample for the initial frame $(0.3 \mathrm{~s})$ and after $0.8 \mathrm{~s}, 1.3 \mathrm{~s}, 1.8 \mathrm{~s}$ and $2.1 \mathrm{~s}$.

The local temperature of the brake disc exceeded $200^{\circ} \mathrm{C}$ after $0.3 \mathrm{~s}$ of braking, thus falling into the preset camera measurement range. It was the beginning of the analyzed sequence of thermal images, which ended as the recorded temperatures exceeded camera's high temperature threshold. At the beginning there are small, high-temperature areas visible in the recorded frames. Those are areas where contact between the friction pair occurred first (and consequently heat emission). The area of temperature analysis (determination of $T_{\max }$ and $T_{\text {mean }}$ values) is marked as the black rectangle, whose longer edge is perpendicular to the direction of disc rotation. Such orientation reduces the temperature averaging (due to the movement of the observed object) on the detector during integration time. The other dimension of the selected area of analysis (rectangle) is much smaller to avoid temperature averaging over a large observed surface. Thermal images were not acquired synchronously with the disc rotation. As a result different areas of the brake disc are visualized on each of them, and sometimes the slot area with much lower temperature can be observed, as on the image recorded for the time of 1.8 second. Those data can be easily discarded in further analyses as they exhibit mean temperature from the range $200^{\circ} \mathrm{C} \leq T_{\text {Mean }} \leq 230^{\circ} \mathrm{C}$. The rise of mean temperature recorded during the braking process is presented in Fig. 9.

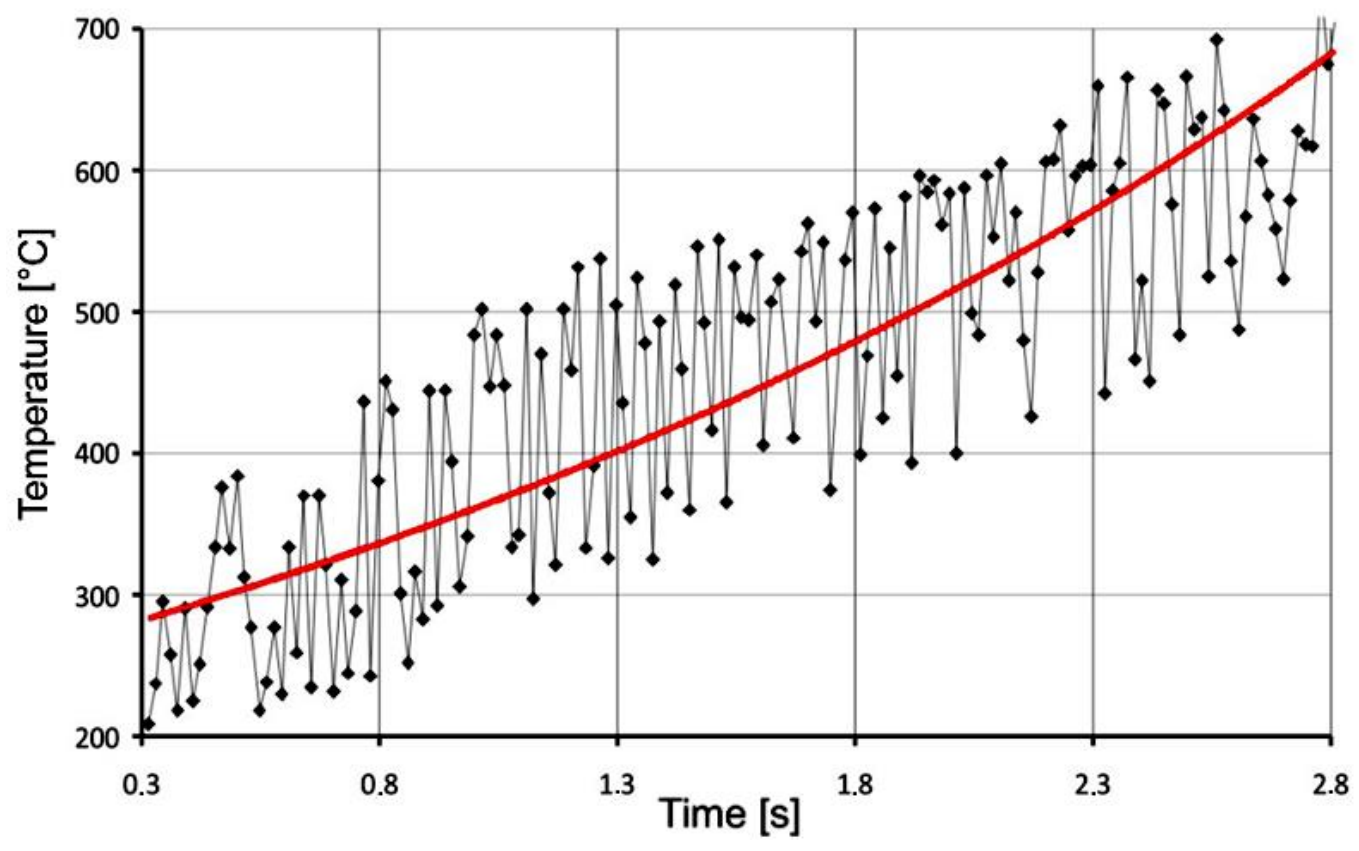

Fig. 9. Temperature changes vs. time inside the brake. 
The time values are given from the beginning of the braking cycle. From 0 to 0.3 second the temperature is too low for the camera to be properly measured (below $200^{\circ} \mathrm{C}$ ). The thermal camera is able to record data up to approximately 2.8 seconds, because after that time the disc temperature exceeds the camera range, detectors get saturated and the measurement results are erroneous. There are large fluctuations of the temperatures recorded on the surface of the friction pair. This is connected with the measurement method itself as well as with tribological effects in the analyzed mechanical node. But in general the measurement results are consistent with the theoretical analysis of mechanical braking. It is also possible to determine the parameters required for numerical modeling of the process, such as maximal temperature values and temperature rise rate, which are directly related to thermal constants characterizing the process. The regression curve indicating a temperature rise is shown in Fig. 9. On this basis the average temperature rise rates can be determined. At the beginning of braking the temperature rises at a rate of $198^{\circ} \mathrm{C} / \mathrm{s}$, and later on, as the areas of interacting surfaces involved in braking get larger, it decreases to $110^{\circ} \mathrm{C} / \mathrm{s}$.

\section{Comparison of results}

A discussion of results can be performed on at least two levels. It can be started from a global overview of the shape of worn samples. Comparing it to the stress distribution obtained from numerical analyses, it can be seen that the most damaged zone of the sample is placed within the area of highest stresses calculated by Finite Element analyses (see Fig. 10). The fact that the stress concentration zone is placed on the edge of the sample is also well explained by contact theory [16]. This indicates that numerical solution is generally consistent.

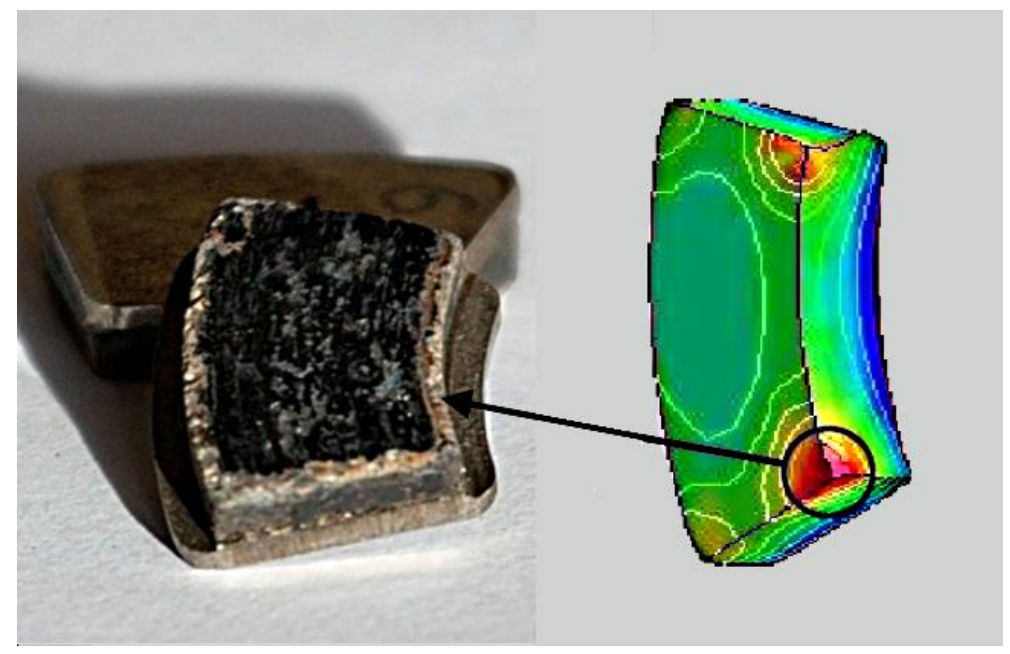

Fig. 10. Comparison of analytically calculated and the actual area of highest stress in the sample material.

Fig. 11 shows temperatures obtained from the IR camera as well as calculated by FE analysis. This chart allows us to move to the next level of results discussion, where more detailed aspects of the analysis can be checked. The curve showing numerical results was obtained by averaging the temperature data taken from one of the samples. Averaging was done to keep consistency with post-processing done by the IR camera software. It can be seen that between $t=0.4 \mathrm{~s}$ and $t=1.4 \mathrm{~s}$ the difference between moving average of measured and calculated temperatures is not bigger than $50^{\circ}$, which all in all can be considered as a reasonable result. 


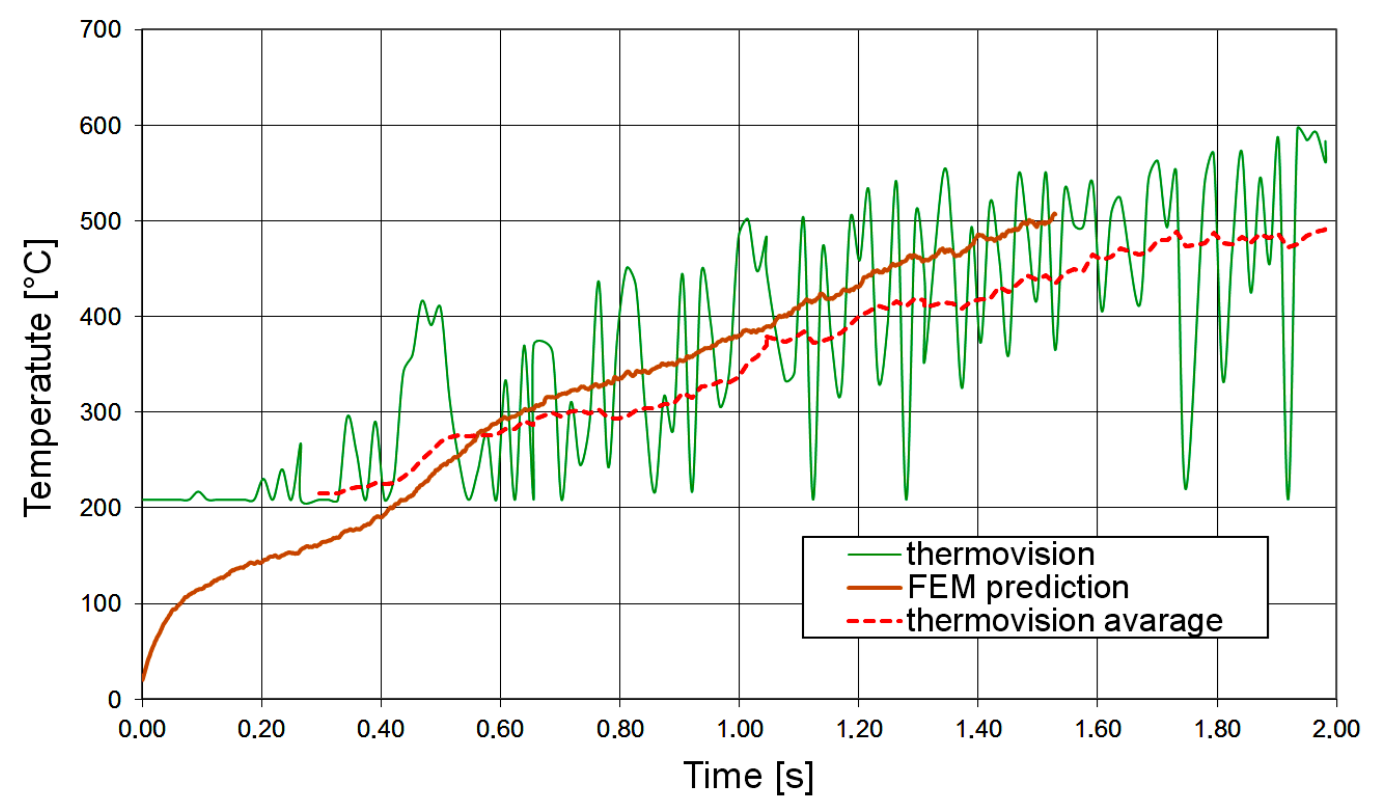

Fig. 11. Comparison of analytically calculated and the actual area of the highest stress in the sample material.

Taking into account that at the same time the temperature rose from about $200^{\circ}$ to $400^{\circ}$, we can say that the difference is less that $20 \%$. For simple, static analyses of mechanically loaded structures such a result is not very impressive. But in the discussed case, where far more complicated phenomena are governing the measured item and even a measurement process is not easy to perform, the obtained correlation is satisfactory.

A very important observation which comes from the graph is that for the first second of braking, the omission of conductivity was indeed acceptable. At the same time it should be noted that the trend of the numerical curve shows that the above may not be true for the very next period of braking. Unfortunately, as it was mentioned, the presented FE model was too demanding in terms of hardware resources to cover a longer braking period.

Finally, the discussion of results should be brought to the level corresponding to the original purpose of the presented FE analyses: wear process simulation. From this perspective, the obtained results are only partially useful. It was confirmed that temperature growth during braking is really very rapid and thus a solution using the explicit time integration scheme was the right choice. The obtained results point out that the models of friction, thermo-mechanical coupling and conversion of mechanical work into heat used in numerical simulations are all working properly on a macroscopic level. The only nonverified algorithm needed to model the actual wear is material erosion, but the inclusion of this phenomenon would require a change of the modeling scale. As it was mentioned, wear takes place on the microscopic level and thus the FE model should be more detailed. This would make such model simply unusable. What is more, the presented validation process was also performed on a global scale and does not give an answer whether the local phenomena are described properly.

\section{Summary}

The braking process and associated lining wear are complex phenomena. An approach to model it using numerical methods requires advanced procedures and models combining several numerical techniques. Validation of such models has to be started by careful choice of validation parameters. In the article the authors showed an approach to deal with one of such processes. Difficulties and labor intensity to measure as well as to analyze the discussed 
phenomenon show that the validation process can be very challenging and close cooperation between those who perform experiments and analyses is essential.

Comparison of experimental and computed data chosen as a validation parameter showed good correlation. Unfortunately, very long analysis run-times did not allow full assessment of analytical model compliance. The overall outcome of the validation process can be summarized as follows: although chosen modelling tools and algorithms work accurately, the numerical model is simply not efficient and it has to be changed in order to cover a longer braking period.

\section{References}

[1] Baranowski, P., Malachowski, J., Mazurkiewicz, L., Kajka, R. (2010). Numerical model of the special test rig called IL-68. Gornictwo odkrywkowe, 3, 93-98.

[2] Kenedy, F.E. (1984). Thermal and thermomechanical effects in dry sliding. Wear, 100, 453-476.

[3] Damaziak, K., Malachowski, J. (2012). Comparison of contact algorithms in the aspect of heat generation in a coupled thermo-mechanical analysis. Przeglad Mechaniczny, 7/8, 15-20.

[4] Oreskes, N., Shrader-Frechette, K., Kenneth Belitz, K. (1994). Verification, validation, and confirmation of numerical models in the earth sciences. Science, New Series, 263(5147), 641-646.

[5] Stolarski, T.A. (1990). Tribology in Machine Design, Butterworth-Heinemann, Oxford.

[6] Eriksson, M., Staffan, J. (2000). Tribological surfaces of organic brake pads. Tribology Int., 33, 817-827.

[7] Renouf, M., Massi, F., Fillot, N., Saulot, A. (2011). Numerical tribology of a dry contact. Tribology Int., 44, 834-844.

[8] Popova, V.L., Psakhie, S.G. (2007). Numerical simulation methods in tribology. Tribology Int., 40, 916-923.

[9] Lodygowski, A., Voyiadjis, G.Z., Deliktas, B., Palazotto, A. (2011). Non-local and numerical formulations for dry sliding friction and wear at high velocities. Int. Journal of Plasticity, 27, 1004-1024.

[10] Meng, H.C., Ludema, K.C. (1995). Wear models and predictive equations: their form and content. Wear, 181/183, 443-457.

[11] Noyes, R.N., Victor, P.T. (1969). Prediction of surface temperatures in passenger car disc brake. $S A E$ technical paper, No. 690457

[12] Sheridan, D.C., Kutchey, J., Samie, F. (1988). Approaches to the thermal modeling of disc brake. SAE technical paper, No. 880256.

[13] Adamowicz, A., Grzes, P. (2011). Influence of convective cooling on a disc brake temperature distribution during repetitive braking. Applied Thermal Engineering, 31, 2177-2185.

[14] Madura, H., Kastek, M., Piatkowski, T. (2007). Automatic compensation of emissivity in threewavelength pyrometers. Infrared Physics and Technology, 51(1), 1-8.

[15] Piatkowski, T., Polakowski, H., Kastek, M., Baranowski, P., Damaziak, K., Malachowski, J., Mazurkiewicz, L. (2012). Thermal measurement of brake pad lining surfaces during the braking process. In Proc. SPIE 8354, $83540 \mathrm{E}$.

[16] Dundurs, J., Lee, M. S. (1972). Stress concentration at a sharp edge in contact problems. Journal of Elasticity, 2(109), 109-112. 